\section{Medizinische Gutachten}

Riemer-Kafka Gabriela (Hrsg.)

Versicherungsmedizinische Gutachten.

Ein interdisziplinärer juristisch-medizinischer Leitfaden

Muttenz: EMH Schweizerischer

Ärzteverlag; 2007 (in Kooperation mit

Stämpfli Verlag AG Bern)

64 Seiten, broschiert.

ISBN: 978-3-03754-003-9

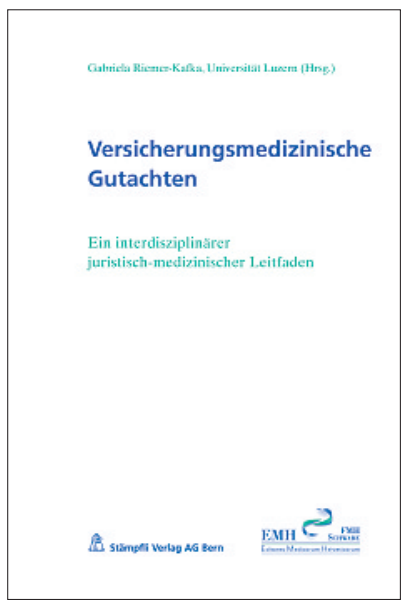

Im Anschluss an eine 1. Tagung des Luzerner Zentrums für Sozialversicherung zum Thema «Medizinische Gutachten» im Jahr 2004 hatten sich Juristen und Mediziner (lic. iur. Yvonne Bollag, Dr. med. Ulrike HoffmannRichter, Dr. med. Jörg Jeger, Prof. Dr. med. Rudolf Kissling, PD Dr. Dr. med. Arnulf Möller, Dr. med., MPH, Ines Rajower, Prof. Dr. iur. Gabriela Riemer-Kafka, Dr. iur. Rudolf Rüedi, Dr. med. Bruno Soltermann, lic. iur. Reto von Steiger, Dr. med. Hans Rudolf Stöckli, Dr. med. Annette Thommen und Prof. Dr. med. Ambros Uchtenhagen) zu einer Arbeitsgruppe zusammengefunden. Deren Ziel bestand darin, zum einen gesprächsweise offene Fragen und vorhandene Verständnisschwierigkeiten zwischen Juristen und Medizinern aufzudecken und zum andern, durch Diskussion eine Annäherung und Abstimmung der gegenseitigen Erwartungen in Bezug auf die Qualität medizinischer Gutachten zu schaffen. Die aus dieser Zusammenarbeit gewonnenen Erkenntnisse werden nun in Form eines Leitfadens für versicherungsmedizinische Gutachten zugänglich gemacht.

Das erste Kapitel des Leitfadens stellt die Funktion medizinischer Gutachten sowie die Aufgaben des ärztlichen Gutachters vor. Die ArztPatienten-Beziehung, die Methodik der Begutachtung und die Voraussetzungen, denen der Gutachter sowie der vom Gutachter verfasste schriftliche Bericht zu genügen haben, werden zunächst aus medizinischer Optik thematisiert. Der nachfolgende juristische Teil erläutert zunächst, in welchen rechtlichen Beziehungen Patient, Entscheidungsträger (Gericht bzw. Verwaltung) und Gutachter zueinander stehen. Sodann werden die durch die Rechtsprechung des Bundesgerichts geprägten Leitlinien zum Beweiswert sowie zur Beweiswürdigung medizinischer Gutachten präsentiert. Abschliessend gelangen die durch diese Rechtsprechung geprägten fachlichen Anforderungen sowohl an den medizinischen Experten als auch an das von ihm verfasste Gutachten zur Darstellung.

Das zweite Kapitel geht näher ein auf spezifische Modalitäten eines versicherungsmedizinischen Gutachtens. Anhand von Beispielen wird dargestellt, mit welchen Fragestellungen die medizinischen Experten häufig konfron- tiert sind. Daran schliessen sich Erörterungen zu zeitlichen Aspekten der Begutachtung sowie zur optimalen Vorgehensweise bei fremdsprachigen Exploranden an. Ausführlich werden Fragen des Datenschutzes behandelt. Abschliessend werden Vorgaben zur formalen und inhaltlichen Gliederung eines Gutachtens formuliert.

Sowohl die Versicherungsmedizin als auch das Versicherungsrecht kennen eine Reihe zentraler Begriffe. Der Leitfaden handelt eine Auswahl davon in seinem letzten Kapitel jeweils aus der medizinischen und der juristischen Perspektive ab. Einige Termini sind sowohl der Jurisprudenz als auch der medizinischen Wissenschaft geläufig, aber je fachspezifisch zu deuten, wie Krankheit, Arbeitsunfähigkeit, Dauerhaftigkeit und Komorbidität. Anderen kommt ein primär rechtlicher Gehalt zu, wie Zumutbarkeit, Erwerbsunfähigkeit, Invalidität und Schadenminderungspflicht.

Die Leser gleich welcher Fachrichtung werden aus der Lektüre des Leitfadens grossen Nutzen ziehen: Insbesondere das letzte Kapitel bildet recht eigentlich eine interdisziplinäre «Übersetzungshilfe». Darin liegt ein sehr wertvoller Beitrag zur Vermeidung von Verständnisschwierigkeiten bereits bei der Formulierung der Expertenfragen, der daran anschliessenden Durchführung der Begutachtung und schliesslich der Lektüre des Gutachtenstextes. Das Werk regt zugleich dazu an, sich mit der Denkweise der jeweils anderen Disziplin näher zu befassen. Dadurch wird es dem Anliegen der Autoren, den Lesern ein Mittel in die Hand zu geben, sich im anderen Fachgebiet kompetent zu bewegen, in hohem Masse gerecht.

lic. iur. H. Dikenmann, Basel 